\title{
Chronic eosinophilic leukaemia: a case report
}

\author{
K.V. Sreedhar Babu, ${ }^{1}$ A.K. Chowhan, ${ }^{2}$ N. Rukmangadha, ${ }^{2}$ B. Vengamma, ${ }^{3}$ M. Kumaraswamy Reddy $^{2}$ \\ Departments of ${ }^{1}$ Immuno Haematology \& Blood Transfusion, ${ }^{2}$ Pathology and ${ }^{3}$ Neurology \\ Sri Venkateswara Institute of Medical Sciences, Tirupati.
}

\begin{abstract}
Chronic eosinophilic leukaemia (CEL) is a rare myeloproliferative disorder of unknown etiology characterized by an autonomous, clonal proliferation of eosinophilic precursors resulting in persistently increased number of eosinophils in the peripheral blood and bone marrow. There is vaguely overlapping clinico - pathological picture of CEL with idiopathic hypereosinophilic syndrome (HES) which often adds to the diagnostic confusion. An evidence of genetic clonality of eosinophils or an increase in blast cells in the blood or bone marrow is mandatory for diagnosis of CEL, while no specific diagnostic tests exist for HES; making it an entity of exclusion. A 41-year old male patient presented with low grade fever associated with drowsiness and heaviness of head since four days and sudden onset of weakness of left lower limb was subjected for complete haemogram followed by bone marrow examination in addition to routine biochemical and radiological evaluation. The peripheral smear and bone marrow aspirates were dominated by the presence of eosinophilic precursors with striking presence of eosinophiloblasts associated with eosinophilic myelocytes, metamyelocytes, a few myeloblasts and basophils compromising erythroid and megakaryocytic elements, the features of which are in favour of CEL. Till date, CEL is a rarely reported entity from India and its presentation with neurological manifestations is still rare. We add yet another case of CEL along with review of available literature.
\end{abstract}

Key words: Chronic eosinophilic leukemia), Idiopathic hypereosinophilic syndrome, Neurological manifestations

Sreedhar Babu KV, Chowhan AK, Rukmangadha N, Kumaraswamy Reddy M. Chronic eosinophilic leukaemia: a case report. J Clin Sci Res 2012;1;46-8.

\section{INTRODUCTION}

Chronic eosinophilic leukaemia is a rare type of chronic myeloproliferative disorder of unknown etiology with no available true incidence. Reliable data on the frequency of CEL do not yet exist and the true incidence is still unknown ${ }^{1}$ owing to the dilemma in distinguishing CEL from HES. However, according to available data, the incidence is highest in the fourth decade of life and the disease most commonly affects males ${ }^{1}$. To make a diagnosis of CEL there should be evidence for clonality of the eosinophils or an increase in blasts in peripheral blood and bone marrow. In CEL, the eosinophil count is greater than or equal to $1.5 \times 10^{9} / \mathrm{L}$. The rarity of CEL is further compounded by a variety of other disease processes accompanied by chronically persistent eosinophilia. Here we intend to present this rare entity who presented clinically with neurological manifestations.

\section{CASE REPORT}

A 41-year-old male patient presented with low grade fever associated with drowsiness and heaviness of head since four days an sudden onset of weakness of left lower lim since two days. He was not a smoker, alcoholic drug addict, did not have any history of allergic disease and was not exposed to any toxins/pesticides. Examination revealed mild pallor with hepatosplenomegaly. Temperature was $101^{\circ} \mathrm{F}$ and pulse rate was $100 /$ min with normal rhythm. The patient was subjected for neurological, hematological, biochemical and radiological assessment. Neurological assessment showed bilateral minimal ptosis with facial weakness, sluggishly reacting pupils, nystagmus on abduction of left eye, hypotonia (power of $2 / 5$ to 3/5) of left lower limb and bilateral brisk knee jerk. Plantars were extensors on left side but flexors on right side (Babinski's sign positive). Sensations are normal all over the body.

Received: 23 December, 2011.

Corresponding author: Dr Kinnera Vijay Sreedhar Babu, Associate Professor, Department of Immuno Haematology \& Blood Transfusion, Sri Venkateswara Institute of Medical Sciences, Tirupati 517 507, India. e-mail: kinneravsb@rediffmail.com 
Haematological investigations have revealed haemoglobin $12.2 \mathrm{~g} / \mathrm{dL}$, total leukocyte count $1,37,500 / \mathrm{mm}^{3}$ with an absolute eosinophil count of $74,550 / \mathrm{mm}^{3}$; the platelet count was $64,000 / \mathrm{mm}^{3}$. The differential counts showed abnormally increased eosinophils with its precursors (83\%), myeloblasts $(8 \%)$, myelocytes $(5 \%)$, metamyelocytes (2\%), neutrophils (1\%) and basophils $(1 \%)$. The reticulocyte count was $2 \%$ and erythrocyte sedimentation rate (ESR) was $15 \mathrm{~mm}$ at the end of the $1^{\text {st }}$ hour hour. Peripheral smear examination revealed mild anemia of normocytic, normochromic type, leukocytic series with good number of dysplastic eosinophils, eosinophiloblasts, myeloblasts, granulocytic precursors and thrombocytopenia (Figure 1). Bone marrow aspirates were hyper cellular dominated by eosinophilic precursors with striking presence of eosinophiloblasts eosinophilic myelocytes, metamyelocytes, a few myeloblasts and basophils compromising erythroid and megakaryocytic elements (Figure 2). Serum electrolytes and liver function tests were within normal limits. Among renal function tests blood urea was $60 \mathrm{mg} / \mathrm{dL}$ where as the serum creatinine was within normal limits. Stool examination was negative for parasitic ova, cysts or blood cells. Serology was negative for Hepatitis $\mathrm{C}$ and human immunodeficiency virus (HIV). Computed tomography (CT) of the brain revealed diffuse cerebral oedema. Basing on these findings a diagnosis of chronic eosinophilic leukaemia was made.

The patient's general condition suddenly

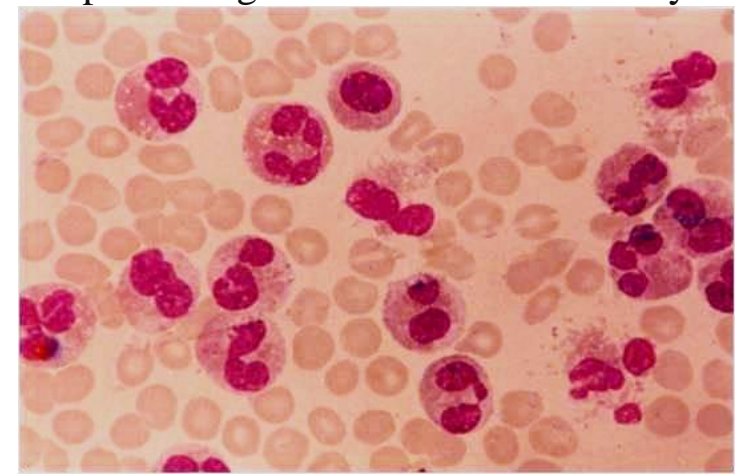

Figure 1: Photomicrograph of peripheral blood smear showing dysplastic eosinophils and its precursors (Leishman $\times 1000)$ deteriorated with systemic bleeding manifestations and loss of sensations which Were managed symptomatically and showed minimal improvement in the level of sensorium that lasted for 24 to 48 hours but he expired on seventh day of admission.

\section{DISCUSSION}

To make a diagnosis of chronic eosinophilic leukaemia, there should be evidence for clonality of the eosinophils or an increase in blasts in the blood or bone marrow (persistent eosinophilia $\geq 1.5 \times 10^{9} / \mathrm{L}$ in blood, increased bone marrow eosinophils more than five percent but less than 19 percent myeloblasts in the bone marrow or more than two percent in the peripheral blood and clonality of myeloid cells). ${ }^{2}$ In many cases it is impossible to prove clonality of the eosinophils. When there is no increase in bone marrow eosinophiloblasts then the diagnosis of idiopathic hypereosinophilic syndrome is preferred. Due to difficulty in distinguishing chronic eosinophilic leukaemia (CEL) from hypereosinophilic syndrome (HES), the true incidence of CEL is unknown, although it is of rare entity. There have been several major reviews in recent years with newly emerging criteria in an attempt to define CEL. Some of the earlier classic reviews relied mainly on the clinico-morphologic attributes of the entity. Evidently, CEL lies on one pole of the continuum of the hypereosinophilic states and may arise from either a multipotent,

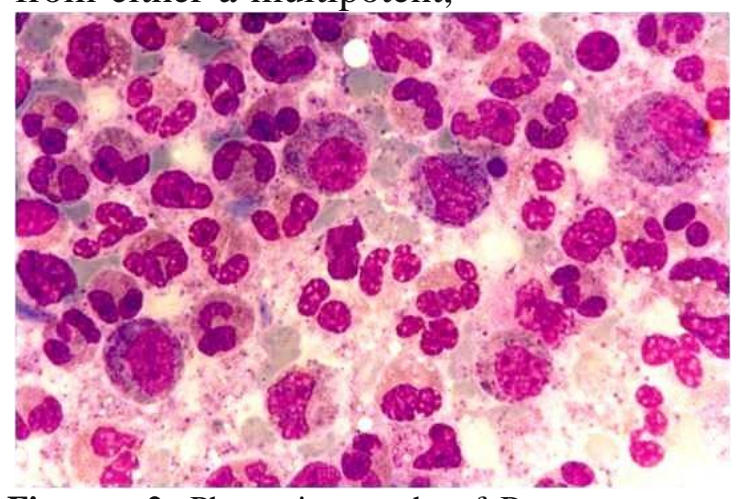

Figure 2: Photomicrograph of Bone marrow aspiration showing dysplastic eosinophilic lineage compromising erythroid and megakaryocytic components (Leishman $\times 1000$ ) 
pluripotent or eosinophil committed progenitor cell. ${ }^{1}$ The eosinophils as part of the neoplastic clone may show a spectrum of characteristic morphological features including marked dysplasia manifested as trilobed or ring formed nuclei, marked cytoplasmic vacuolation, hyper or hypogranulation and small or "microeosinophils". Some may even show basophilic and eosinophilic granulations together. The neoplastic, monoclonal nature of eosinophils has been further substantiated by various cytogenetic studies showing a multitude of chromosomal abnormalities especially trisomy 15 , trisomy $8,{ }^{3}$ isochromosome 17 , translocations $\mathrm{t}(2 ; 5)(\mathrm{p} 23 ; \mathrm{q} 31)^{4}$ and $\mathrm{t}(5$; 12) (q33; p13) and molecular genetic abnormalities particularly linked to eosinophil differentiation (such as formation of a FIP1L1- PDGFRA fusion gene). ${ }^{5}$

Almost all reported cases in Indian subcontinent belong to hypereosinophilic syndrome; ${ }^{6}$ majority of them being associated with parasitic involvement or allergic respiratory diseases. Our case meets the morphologic criteria of CEL. Though the karyotype could not be investigated, a presumptive diagnosis of eosinophilic leukemia is justified in view of the markedly high absolute eosinophil count. Besides, the characteristic morphology showing obvious proliferation of eosinophilic precursors along with dysplastic features confirms the neoplastic nature of eosinophils as against hypereosinophilic syndrome where the increased eosinophilic population is largely reactive. Survival usually depends on the quantity of eosinophiloblasts at the onset of the disease and rapidly declines with increasing numbers of eosinophiloblasts. Our case study is an attempt to highlight the need for caution in diagnosing patients with unexplained eosinophilia, even when conclusive cytogenetic proof of the neoplastic nature of the disease is lacking. Till date, CEL is not a well-defined entity, and differentiation between CEL and HES can be challenging. In many instances, a correct diagnosis can only be established after serial observations and investigations over time. The multiorgan damage and rapidly aggressive clinical course in such patients resulting from release of cytokines and other enzymes from eosinophilic granules (major basic protein and eosinophilic cationic protein) as well as leukaemic tissue infiltration calls for prompt identification and institution of therapy. The optimal treatment for CEL remains unclear due in part to the rarity of this chronic myeloproliferative disorder and the variable clinical course, which can range from cases with decades of stable disease to those with rapid progression to acute leukaemia. Case reports suggest that treatment options include bone marrow transplantation and interferon- $\alpha$.

The present case is being reported not only because of its rarity but also because of its interesting clinical presentation.

\section{REFERENCES}

1. Vardiman JW: Myelodysplastic myeloproliferative diseases: introduction. In: Jaffe ES, Harris NL, Stein H, et al., eds.: Pathology and Genetics of Tumours of Haematopoietic and Lymphoid Tissues. Lyon, France: IARC Press, 2001. World Health Organization Classification of Tumours, 3, pp 47-8. 2. Bain B, Pierre P, Imbert M, et al.: Chronic eosinophillic leukaemia and the hypereosinophillic syndrome. In: Jaffe ES, Harris NL, Stein H, et al., eds.: Pathology and Genetics of Tumours of Haematopoietic and Lymphoid Tissues. Lyon, France: IARC Press, 2001. World Health Organization Classification of Tumours, 3, pp 2931.

3. Ma SK, K Y, Shek TW, Wan TS, Chow EY, Chan JC, Chan LC. The role of trisomy 8 in the pathogenesis of chronic eosinophilic leukemia. Hum Pathol 1999;30:864-8.

4. Lepretre S,JardinF, Buchonnet G, Lenain P, Stamatoullas A, Kupfer I,et al. Eosinophilic leukemia associated with $\mathrm{t}(2 ; 5)(\mathrm{p} 23 ; \mathrm{q} 31)$. Cancer Genet $\mathrm{Cy}-$ togenet 2002;133:164-7.

5. Cools J, DeAngelo DJ, Gotlib J, Stover EH, Legare RD, Cortes J, et al. A tyrosine kinase created by fusion of the PDGFRA and FIP1L1 genes as a therapeutic target of imatinib in idiopathic hypereosinophilic syndrome. N Engl J Med 2003;348:1201-14.

6. Venkatesh CME, Janani S, Malathi S, Vijayakumar M, Nammalwar BR. Hypereosinophilic syndrome. Indian J Pediatr 2006;73:237-9. 